been proposed as the location of the probe molecule, based on the chemical shift in 'H NMR measurements. ${ }^{15}$

On the other hand, there has been a suggestion that water can penetrate into micelles. ${ }^{16}$ If this is the case, the amount of the penetration is expected to be pressure dependent. In our results, however, the $I_{1} / I_{3}$ ratio for micellar state was almost constant within our experimental errors. This may indicate that the water penetration is constant and limited in the neighborhood of the polar head groups. But this aspect needs to be examined further.

The pressure dependence of the $\mathrm{cmc}$, which is shown in Figure 7 , is connected with the change in the partial molal volume in the micelle formation by the following equation: ${ }^{17}$

$$
(1+\beta) R T\left\{\frac{\partial \ln (\mathrm{cmc})}{\partial P}\right\}_{T}=\Delta \bar{V}_{\mathrm{m}}
$$

$\Delta \bar{V}_{\mathrm{m}}=\bar{V}_{\mathrm{m}}-\bar{V}_{\mathrm{s}}$, where $\bar{V}_{\mathrm{m}}$ and $\bar{V}_{\mathrm{s}}$ are the partial molal volumes of the surfactant in the micellar and singly dispersed states, respectively. $\beta$ is the number ratio of counterion to surfactant ion in micelles. By neglecting the variation of $\beta$ with pressure, the value of $\Delta \bar{V}_{\mathrm{m}}$ at atmospheric pressure, calculated from initial slope of the pressure dependence (Figure 4 ), is $+13 \mathrm{~cm}^{3} / \mathrm{mol}$, when a value of 0.73 is adopted for $\beta .^{18}$ This plot was satisfactorily represented as a polynomial of third degree in pressure.

The appearance of a maximum appeared in the plot of $\mathrm{cmc}$ against pressure indicates that $\Delta \bar{V}_{\mathrm{m}}$ changes its sign from positive to negative. The positive volume change of micellization has been attributed to the elimination of hydrocarbon-water contact accompanied with micellization. ${ }^{8}$ But why does it change its sign with pressure? Is it simply because the micellar state is much

(15) Zachariasse, K. A.; Kozankiewicz, B.; Kühnle, W. Surfactant in Solution; Mittal, K. L., Fendler, F. J., Eds.; Plenum: New York, 1984; Vol. $1, \mathrm{p} 565$.

(16) Fendler, J. H.; Fendler, E. J. Catalysis in Micellar and Macromolecular Systems; Academic Press: New York, 1975.

(17) Tuddenham, R. F.; Alexander, A. E. J. Phys. Chem. 1962, 66, 1839. more compressible than the singly dispersed state, as has been suggested ${ }^{18}$

The unexpected decrease of the apparent micropolarity in nonmicellar region as seen in Figure 5 seems to have something to do with this problem. In other words, the decrease in micropolarity of the singly dispersed state can be caused by some pressure-induced aggregation around the probe molecule due to hydrophobic interaction, which might be called microemulsion. This process is expected to be accompanied with an increase in the $\bar{V}_{\mathrm{s}}$ value with pressure. In fact, a negative value of apparent molal compressibility for the single dispersed state has been reported. ${ }^{8}$ Therefore, $\bar{V}_{\mathrm{s}}$ rather than $\bar{V}_{\mathrm{m}}$ may predominantly contribute to the change in the sign of $\Delta \bar{V}_{\mathrm{m}}$.

In the results of the present work, the $\mathrm{cmc}$ values defined from the terminal point 2 agreed well with those from conductivity method, although in the paper of Turro et $\mathrm{al}^{4}$ the inflection point was taken as the $\mathrm{cmc}$. The occurrence of the substantial transition region in the $I_{1} / I_{3}$ value in the fluorescence technique may suggest the preliminary appearance of the aggregates with lower aggregation number, as has been called "premicelle" state, ${ }^{19}$ prior to the formation of ordinary stable large micelles. The higher the pressure is, the broader the transition becomes. It would also be closely correlated with the discussion mentioned above.

From the point of view of a high-pressure study not only for the cmc determination but also for the investigation of various microproperties of micelle interiors, we can conclude that the fluorescence probe technique is a useful and reliable method.

Acknowledgment. We thank Prof. Y. Taniguchi of Ritsumeikan University for the help and cooperation throughout this work.

Registry No. SDS, 151-21-3; pyrene, 129-00-0.

(18) Kaneshina, S.; Tanaka, M.; Tomida, T. J. Colloid Interface Sci. 1974, 48,450 .

(19) Somasundaran, P.; Ananthapadmanabhan, K. P.; Ivanov, I. B. J. Colloid Interface Sci. 1984, 99, 128.

\title{
Surface-Enhanced Resonance Raman Scattering from Langmuir-Blodgett Monolayers: Surface Coverage-Intensity Relationships
}

\author{
Jae-Ho Kim, Therese M. Cotton, ${ }^{*}$ R. A. Uphaus, \\ Department of Chemistry, University of Nebraska-Lincoln, Lincoln, Nebraska 68588-0304
}

and D. Möbius

Max-Planck Institut für biophysikalische Chemie, D-3400 Göttingen-Nikolausberg, FRG (Received: August 16, 1988)

\begin{abstract}
The relationship between surface coverage and surface-enhanced resonance Raman scattering (SERRS) was examined for a cyanine dye (S-120) dispersed in Langmuir-Blodgett monolayers containing arachidic acid and methyl arachidate as an inert diluent. The monolayers were supported on $\mathrm{Ag}$ island films that were vacuum-deposited on glass slides. The optical properties (absorbance/transmittance) and excitation profiles of the SERRS intensities were also measured as a function of concentration. The maximal SERRS enhancement was observed at $500 \mathrm{~nm}$ or close to the Ag plasmon resonance of the films. The results show that SERRS intensities for various vibrational modes of the dye maximize at submonolayer dye coverage. The observed nonlinear coverage behavior is attributed to changes in the dielectric properties and/or dipole-dipole coupling between the dye molecules as a function of surface coverage.
\end{abstract}

\section{Introduction}

The enormous sensitivity resulting from the surface-enhanced Raman effect suggests that it should provide a powerful technique for surface analysis. However, as indicated by the considerable numbers of experimental and theoretical papers that have appeared during the past 10 years, there are many uncontrolled or poorly defined variables that can profoundly affect the magnitude of the enhancement. These include the optical properties of the metal substrate (Ag has been the most frequently studied), the morphology of the substrate, the surface potential of the metal, the excitation wavelength used to produce Raman scattering, the metal-adsorbate interactions (chemisorption vs physisorption), the distance between the adsorbate and the metal surface, and the optical properties of the medium surrounding the metal and 
the adsorbate (recent reviews may be found in ref 1 ). Wang and Kerker ${ }^{\text {Id }}$ have developed a detailed theory and performed calculations for the SERS response of spheroids.

The influence of the optical properties of the surrounding medium on the signal intensity is predicted from electromagnetic enhancement (EM) theory. ${ }^{2}$ These properties are dependent, in turn, upon the adsorbate surface coverage. ${ }^{3,4}$ Furthermore, theory predicts that the response of the system can be quite complex when the adsorbate has electronic states close to those of the substrate., 3 For example, excitation profiles of adsorbates that are both surface and resonance enhanced (surface-enhanced resonance Raman scattering, SERRS) are predicted to exhibit splittings in some cases. ${ }^{5}$ Two models for the coverage behavior of resonantly enhanced molecules were recently introduced by Zeman et al. ${ }^{5}$ The layered spheroid model analyzes the optical response of a dyecoated metal spheroid in terms of its dielectric properties. The dielectric properties are approximated by an effective medium approach, which considers both the adsorbate and ambient medium and, hence, are coverage dependent. The coupled dipole model assumes a prolate metal particle surrounded by a submonolayer of dye molecules randomly distributed over available surface sites. Both models predict dramatic effects on the SERRS intensities as a function of surface coverage. Significant changes in the magnitude of the enhancement are also predicted for particular modes as a function of excitation frequency and Stokes shift. The decrease in enhancement for coverages greater than 0.1 monolayer is predicted to arise primarily as a result of damping of the plasmon resonance by the adsorbed layer, rather than from interadsorbate interactions.

There have been only a limited number of experimental systems in which the resolution of SERS, SERRS, and RRS was effected by a comparison of a dye and a nonchromophore on the same substrate. An excellent example of such systems is found in the study of 4-(dimethylamino)azobenzene-4'-sulfonyl aspartate (DABS-ASP) and pyridine by Siiman et al. ${ }^{6}$

Non-Resonance-Enhanced Adsorbates: SERS. Nonlinear signal intensity vs surface coverage behavior was observed in early studies of pyridine on $\mathrm{Ag}$ under ultrahigh-vacuum (UHV) conditions. ${ }^{7-12}$ The advantages to the UHV approach include the capability of preparing a defined surface and an accurate determination of the coverage by Auger electron spectroscopy or other quantitative methods. The goal of the initial studies was focused primarily on proving ${ }^{9,10}$ or disproving 7,8 the existence of long-range enhancement in multilayers. In addition, the unusual relationship between SERS intensities and adsorbate surface coverage was clearly evident in several papers. ${ }^{10-12}$ An increase in pyridine SERS intensity was seen for coverages up to ca. 0.5 monolayer. At higher coverages the intensity decreased and remained constant ${ }^{11}$ or increased slowly. ${ }^{10}$ In one case, ${ }^{8}$ the SERS intensity was shown to peak at ca. 1 monolayer and decrease with increasing coverage. Variations in the reported surface coverage

(1) (a) In Surface Enhanced Raman Scattering, Chang, R. K. Furtak T. E., Eds.; Plenum Press: New York, 1982. (b) Van Duyne, R. P. In Chemical and Biochemical Applications of Lasers, Moore, C. B., Ed.; Academic Press: New York, 1979, Vol. 4, pp $101 \mathrm{ff}$. (c) Cotton, T. M. Adv. Spectrosc. 1988, 16, 91. (d) Wang, D.-S.; Kerker, M. Phys. Rev. B 1981, 24, $1777-1790$.

(2) (a) Metiu, H.; Das, P. Ann. Rev, Phys. Chem. 1984, 35, 507. (b) Moskovits, M. Rev, Mod. Phys. 1985, 57, 783 .

(3) Kotler, Z.: Nitzan, A. J. Phys. Chem. 1982, 86, 2011

(4) Chew, H.; Wang, D.-S.; Kerker, M. Phys. Rev. B 1983, 28,4169

(5) Zeman, E. J.; Carron, K. T.; Schatz, G. C.; Van Duyne, R. P. J. Chem. Phys. 1987, 87, 4189 .

(6) Siiman, O.; Smith, R.; Blatchford, C.; Kerker, M. Langmuir 1985, I, 90-96.

(7) Smardzewski, R. R.; Colton, R. J.; Murday, J. S. Chem. Phys. Lett. $1979,68,53$

(8) Pockrand, L.: Otto, A. Solid State Commun. 1980, 35, 861

(9) Zwemer, D. A.; Shank, C. V.; Rowe, J. E. Chem. Phys. Lett. 1980, 73, 201

(10) Rowe, J. E.; Shank, C. V.: Zwemer, D. A.; Murray C. A. Phys, Rev. Lett. 1980, 44, 1770

(i1) Eesley, G. L. Phys. Lett. 1981, 81 A, 193.

(12) Pockrand, I. Chem. Phys. Lett. 1982, 92, 509. values that exhibit maximal enhancement may be due in part to the differences in substrate preparation and/or the method of dosing the surface with pyridine and determining the surface concentration. Explanations for the nonlinear response included that of Eesley, who proposed that the coverage dependence reflected dipolar interactions between molecules. ${ }^{11}$

There have also been several studies of the effect of surface coverage on SERS intensities under ambient conditions. The SERRS intensity of $\mathrm{CN}^{-}$on $\mathrm{Ag}$ films in air was observed to follow a pattern similar to that described above for pyridine in UHV. ${ }^{13}$ In case of the $\mathrm{CN}^{-}$, two different adsorbed species were identified, on the basis of two distinct $\mathrm{CN}$ stretching frequencies. When the integrated intensities of the two bands were plotted as a function of surface coverage, the intensity peaked at ca. 0.25 monolayer and decreased to about $60 \%$ of the original intensity at 1.00 monolayer coverage. Using classical microscopic local-field calculations that included the electronic polarizabilities of nearby adsorbates, Murray and Bodoff ${ }^{13}$ showed excellent agreement between the predicted and observed coverage dependence. In a subsequent study ${ }^{14}$ the coverage dependence of the two sites was determined separately. The absolute enhancement value was determined, and the chemical enhancement was found to be only ca. $6 \pm 3$, with an electromagnetic enhancement of $10^{4}$ contributing the bulk of the enhancement. The effect of coadsorbates on the surface coverage was also examined. When aromatic hydrocarbon impurities were present at approximately 0.1 monolayer, the intensity of the $\mathrm{CN}$ stretching mode was found to follow the same relative surface coverage dependency, but the absolute intensity was only $70 \%$ of that observed for "clean" substrates. The decrease in scattering intensity was attributed to the depolarizing influence of nearby aromatic adsorbates. More recently, these studies have been extended to include cyanide adsorption on silver and gold overlayers on island films of $\mathrm{Au}$ or $\mathrm{Ag}$, respectively. ${ }^{15}$ A simple model was developed to predict the effects of overlayers or spacers composed of $\mathrm{Au}, \mathrm{Ni}$, and nonabsorbing dielectrics on the EM enhancement of adsorbates on prolate spheroids. ${ }^{16}$

Ag island films were also used in a study of the SERS intensities of $p$-nitrobenzoate as a function of surface coverage by Weitz et al. ${ }^{17}$ The $p$-nitrobenzoate was adsorbed spontaneously from ethanol solutions, and the surface coverage was varied by changing the solution concentration. The SERS intensity was shown to increase monotonically for solutions ranging in concentration from $10^{-7}$ to $10^{-6} \mathrm{M}$. At concentrations greater than $10^{-5} \mathrm{M}$, no increase in SERS intensity was observed. No evidence of anomalous concentration effects are apparent in this data

Resonance-Enhanced Adsorbates: SERRS. The relationship between surface coverage and SERRS intensities has not been studied as extensively as is the case for non-resonance-enhanced species. Most investigators have employed $\mathrm{Ag}$ sols and determined SERRS intensities as a function of dye concentration in the bulk. Some indication of a decrease in enhancement with increasing surface concentration of rhodamine $6 \mathrm{G}$ or basic fuchsin on $\mathrm{Ag}$ island films was noted in the study by Weitz et al. ${ }^{17}$ For both dyes, the enhancement factor was found to decrease by 1 order of magnitude when adsorbed from highly concentrated solutions, as compared to dilute solutions. In a detailed study of the SERRS behavior of rhodamine $6 \mathrm{G}$ on colloidal $\mathrm{Ag}$, Hildebrandt and Stockburger ${ }^{18}$ found a linear relationship between the log concentration of dye in solution and log SERRS intensity. Linear adsorption isotherms were determined in the concentration range between $10^{-11}$ and $10^{-5} \mathrm{M}$. A strong dependence of SERRS intensity on the nature of the anion was also observed. Chloride ion was found to enhance the SERRS intensity by an order of magnitude over iodide ion.

(13) Murray, C. A.; Bodoff, S. Phys. Rev. Lett. 1984, 52, 2273

(14) Murray, C. A.; Bodoff, S. Phys. Rev. B 1985, 32, 671

(15) Murray, C. A.; Bodoff, S. J. Chem. Phys. 1986, 85, 573

(16) Murray, C A J Opt Soc Am B 1985, 2, 1330

(17) Weitz, D. A.; Garoff, S.; Gersten, J. I.; Nitzan, A. J. Chem. Phys. $1983,78,5324$.

(18) Hildebrandt, P.; Stockburger, M. J. Phys. Chem. 1984, 88, 5935. 
Zeman et al..$^{5}$ examined the effect of surface coverage using cobalt phthalocyanine $(\mathrm{CoPc})$ vapor-deposited onto $\mathrm{CaF}_{2}$ roughened silver films. The experimental data were compared with their two theoretical treatments. The importance of accurate surface coverage measurements was stressed in this paper. Quantitation of the adsorbed $\mathrm{CoPc}$ was obtained by washing the CoPc from the film with pyridine and measuring the absorbance of the solution. The data showed a maximum in the SERRS intensity at only 0.067 monolayer. At higher coverages the signal decreased rapidly and remained approximately constant for coverages greater than 2 monolayers. An estimate of the enhancement factor was obtained by comparing the SERRS intensity with the resonance Raman (RR) intensity of $\mathrm{CoPc}$ on a smooth chromium film.

Electronic Absorption/Transmission Spectroscopy. Large changes in the optical properties of the dye-coated $\mathrm{Ag}$ films affect the overall SERRS enhancement. $3,5,16,17,24,33$ Previous investigations have shown that the optical properties (absorption ${ }^{14,19-21,23,24}$ and fluorescence ${ }^{19,22,24-33}$ ) of adsorbate-coated $\mathrm{Ag}$ films are strongly influenced by adsorbate surface coverage. Both non-resonance-14,24 and resonance-enhanced ${ }^{19-21,23}$ adsorbates were studied. With respect to the latter, it was demonstrated experimentally that the absorption spectrum of the composite $\mathrm{Ag}$ film/dye overlayer is strongly dependent upon the overlap between the dye and the $\mathrm{Ag}$ particle plasma resonance. Using a wedge technique, Glass et al. ${ }^{19}$ showed that a splitting in the dye/Ag island spectrum results when the dye and particle absorption maxima are coincident. The splitting was attributed to damping of the metal particle resonance by absorption of the dye coating on the particle. This results in a minimum in the composite spectrum at the maximum absorption wavelength of the dye. Electromagnetic theory has been used successfully to account for the shifts in frequency and splitting in the plasmon frequency that accompany dye adsorption on $\mathrm{Ag}$ particles or island films. ${ }^{3,20,21,23,24}$ The absorption properties of a model system composed of dye-coated metal spheroids has been shown to depend upon the particle size, the optical properties of the core dielectric, the coating thickness, and the spheroid eccentricity. ${ }^{24}$ Thus, the response of the a dye-coated Ag island film is difficult to predict without knowledge of all of these experimental parameters.

Use of Langmuir-Blodgett Monolayers in SERRS Studies. Langmuir-Blodgett (LB) monolayers offer a precise method for preparing chemically and structurally defined systems on surfaces. Previously LB multilayers were used to probe the dependence of SERRS intensity upon the distance of an adsorbate from a silver island film surface. ${ }^{34,35}$ The results confirmed that electromagnetic

(19) Glass, A. M.; Liao, P. F.; Bergman, J. G.; Olson, D. H. Opt. Lett. $1980,5,368$.

(20) Garoff, S.; Weitz, D. A.; Gramila, T. .; Hanson, C. D. Opt. Lett. 1981, 6,245 .

(21) Craighead, H. G.; Glass, A. M. Opt. Lett. 1981, 6, 248.

(22) Garoff, S.; Stephens, R. B.; Hanson, C. D.; Sorenson, G. K. Opt. Commun. 1982, $41,257$.

(23) Eagen, C. F. Appl. Opt. 1981, 17, 3035.

(24) Wang, D.-S.; Kerker, M. Phys. Rev. B 1982, 25, 2433.

(25) Ritchie, G.; Burstein, E. Phys. Rev. B 1981, 24, 4843.

(26) Weitz, D. A.; Garoff, S.; Hanson, C. D.; Gramila, T. J.; Gersten, J. I. J. Luminesc. 1981, 24/25, 83.

(27) Glass, A. M.; Wokaun, A.; Heritage, J. P.; Bergman, J. G.; Liao, P. F.; Olson, D. H. Phys. Rev. B 1981, 24, 4906.

(28) Weitz, D. A.; Garoff, S.; Hanson, C. D.; Gramila, T. J.; Gersten, J. I. Opt. Lett. 1982, 7, 89 .

(29) Wokaun, A.; Lutz, H.-P.; King, A. P.; Wild, U. P.; Ernst, R. R. J. Chem. Phys. 1983, 79, 509 .

(30) Leitner, A.; Lipptisch, M. E.; Draxler, S.; Riegler, M.; Aussenegg, F. R. Appl. Phys. B 1985, 36, 105

(31) Garoff, S.; Weitz, D. A.; Alverez, M. S. Chem. Phys. Lett. 1982, 93, 283

(32) Garoff, S.; Weitz, M. S.; Alverez, M. S.; Gersten, J. I. J. Chem. Phys. $1984,81,5189$

(33) Gersten, J. I.; Nitzan, A. Surf. Sci. 1985, $158,165$.

(34) Cotton, T. M.; Uphaus, R. A.; Möbius, D. J. Phys. Chem. 1986, 90, 6071 .

(35) Kovacs, G. J.; Loutfy, R. O.; Vincett, P. S.; Jennings, C.; Aroca, R. Langmuir 1986, 2, 689.
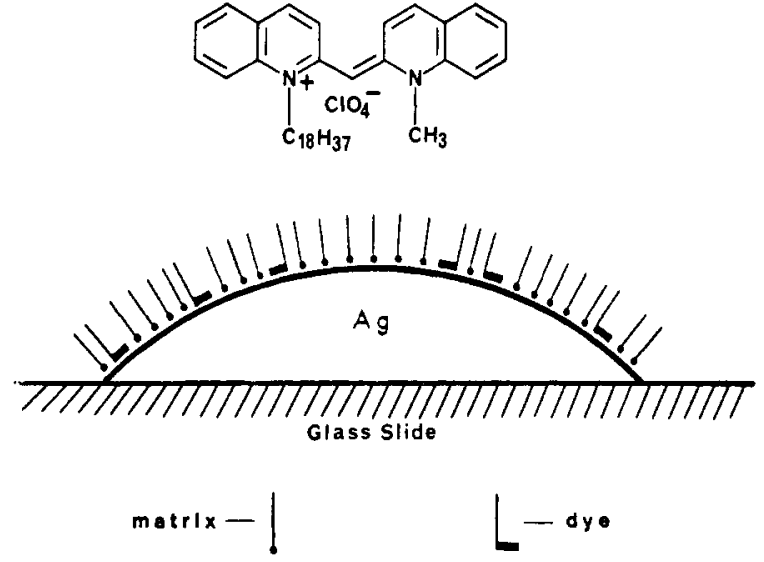

Figure 1. Molecular structure of S-120 (1-methyl-1'-octadecyl-2,2'cyanine perchlorate) and a schematic of the dye-lipid monolayer on $\mathrm{Ag}$-coated glass slide. The dye and lipid representations are shown approximately to the same scale as the Ag particle (ca. $400-\AA$ diameter).

enhancement provides a strong contribution to the overall enhancement process. In the present study, monolayers containing varying concentrations of the dye S-120 (1-methyl-1'-octadecyl-2,2'-cyanine perchlorate) in a lipid matrix (arachidic acid and methyl arachidate) were transferred to silver island films deposited on glass slides. In this manner, it was possible to vary the precise dye concentration from less than 0.5 to $30 \mathrm{~mol} \%$ and the determine the effect of dye coverage on the resulting SERRS intensity.

\section{Experimental Methods}

The S-120 dye was prepared according to literature procedures. $^{36}$ All other chemicals were from commercial sources and were the purest grades available.

Silver island films (5-nm thickness) were deposited at room temperature onto clean glass slides by using a vacuum deposition system. Immediately following the Ag deposition, the slides were coated with monolayers by using standard techniques. ${ }^{37}$ The dipping speed was $1.3 \mathrm{~cm} / \mathrm{min}$, and the surface pressure during transfer was $20 \mathrm{mN} / \mathrm{m}$. The dye concentration in the monolayers was varied between 0.5 and $33.3 \mathrm{~mol} \%$ by using arachidic acid/methyl arachidate (9:1 mole ratio) as the inert diluent. Figure 1 is a schematic of the dye/matrix layer on the Ag-coated glass substrate. The molecular structure of S-120 is also shown. The homogeneity and integrity of the transferred dye monolayers were determined by using the fluorescence energy transfer test described by Kuhn. ${ }^{38}$

Raman spectra were recorded by using the previously described system. ${ }^{39} \mathrm{An} \mathrm{Ar}^{+}$(INNOVA 90-5, Coherent Radiation, Inc.) or a $\mathrm{Kr}^{+}$(INNOVA 100-K3, Coherent Radiation, Inc.) laser was used as an excitation source. The Raman scattered light was collected in the backscattering geometry and focused onto the slits of a spectrometer/spectrograph (Spex, Triplemate 1877). An intensified silicon photodiode array (Princeton Applied Research, Model 1420) served as the detector, and the signals were acquired and processed by using an optical multichannel analyzer (OMA II, Princeton Applied Research). In most cases, the signal was integrated for 60 delays (ca. $1 \mathrm{~s}$ ). Multiple scans are indicated in the figure legends.

Electronic absorption spectra of the dye-containing monolayers on glass slides were recorded as described previously. ${ }^{37}$ The spectra are plotted as the difference in transmission $\Delta T$ vs wavelength between the monolayer-coated slide and an uncoated portion of the slide as the reference, i.e., $\Delta T=(I(\mathrm{R})-I(\mathrm{D})) / I(\mathrm{R})$, where

(36) Sonderman, J. Liebigs Ann. Chem. 1971, 749, 183.

(37) Kuhn, M.; Möbius, D.; Bücher, H. In Physical Methods of Chemistry; Weissberger, A., Rossiter, B., Eds.; Wiley: New York, 1972; Vol. 1, Part 3B.

(38) Kuhn, H. Thin Solid Films 1983, 99, 1.

(39) Holt, R. E.; Cotton, T. M. J. Am. Chem. Soc. 1987, 109, 1841 


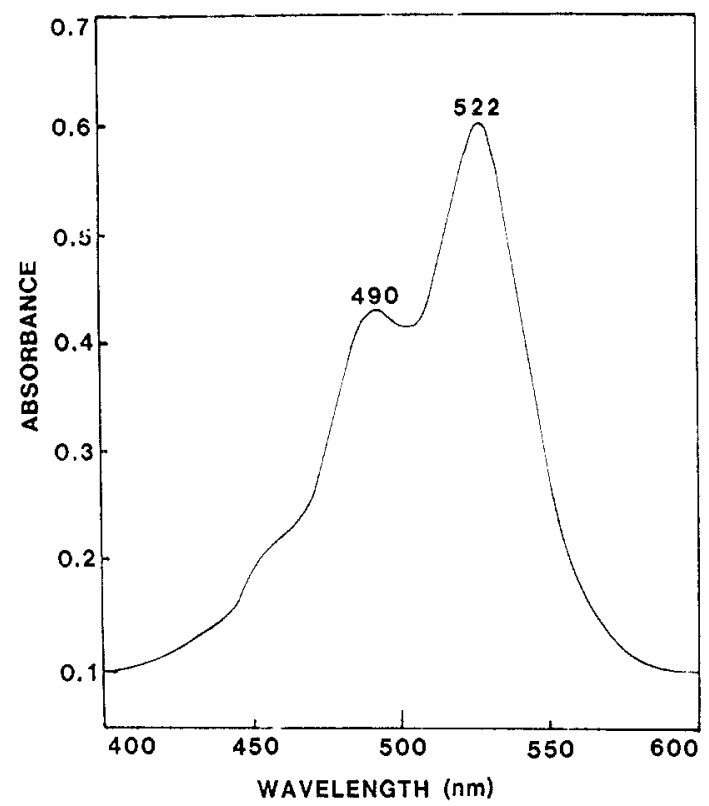

Figure 2. Electronic absorption spectrum of S-120 $\left(3.19 \times 10^{-5} \mathrm{M}\right)$ in methanol.

$I(\mathrm{R})$ and $I(\mathrm{D})$ are the intensities of light transmitted by the reference and sample sections of the slide, respectively. The optical density can be calculated from $\Delta T$ by taking into account the enhanced reflectance of the sample section as compared to the reference section. ${ }^{40}$

\section{Results}

The optical properties of the LB films on Ag-coated glass slides (Figure 1 is a schematic of the system) significantly affect the SERRS response. Therefore, the spectra of the dye in solution, in LB films on glass slides, and in LB films on Ag-coated glass slides are discussed prior to the discussion of SERRS results.

Electronic Absorption and Transmission Spectrum of $S-120$ At the concentration examined $\left(3.19 \times 10^{-5} \mathrm{M}\right)$, the dye is monomeric in methanol solution. As shown in Figure 2, the absorption maximum is at $522 \mathrm{~nm}$ with shoulders near 490 and 460 $\mathrm{nm}$. At higher concentrations or when deposited in LB monolayers, the dye forms J-aggregates. ${ }^{41}$ Figure 3 depicts the $\Delta T$ spectra of single monolayers on glass slides containing increasing concentrations of dye in the lipid matrix. In spectra 3A-D (top; dye concentrations ranging from 1.0 to $7.7 \mathrm{~mol} \%$ ) there are two major bands of approximately equal intensity at 490 and $535 \mathrm{~nm}$. The latter band shifts to the red with increasing coverage. Both bands increase linearly with increasing dye concentration. At 9 mol \% a sharp band near $580 \mathrm{~nm}$ becomes dominant. This band is attributed to the J-aggregate and increases in intensity as the dye concentration increases from 9.0 to $18.2 \%$ (Figure 3F-I, bottom). Note that the scale for the series of spectra on the bottom is 10-fold greater than that on top. Thus, the decrease in transmission accompanying $\mathbf{J}$-aggregate formation is abrupt. Moreover, the J-aggregate band is not linearly related to concentration. As the J-aggregate fraction in the monolayer becomes dominant, the reflectance of the dye, which contributes to the measured $\Delta T$, increases nonlinearly with mole percent.

Electronic Absorption/Transmission Spectrum of the Ag Films and the Composite Dye-Ag Films. The optical properties of the $\mathrm{Ag}$ island films depend strongly upon a number of different parameters, including the nature of the substrate, the film thickness, the rate of deposition, and the temperature of the substrate. ${ }^{42,43}$ The films used in these studies are composed of small, well-sep-

(40) Orrit, M.; Möbius, D.; Urs, L.; Meyer, H. J. Chem. Phys. 1986, 85, 4966.

(41) Scheibe, G. Angew. Chem. 1936, 49,563.

(42) Handbook of Thin Film Technology; Maissel, L. I., Glang, R., Eds.; McGraw-Hill: New York, 1970.

(43) Sennett, R. S.; Scott, G. D. J. Opt. Soc. Am. 1950, 40, 203.
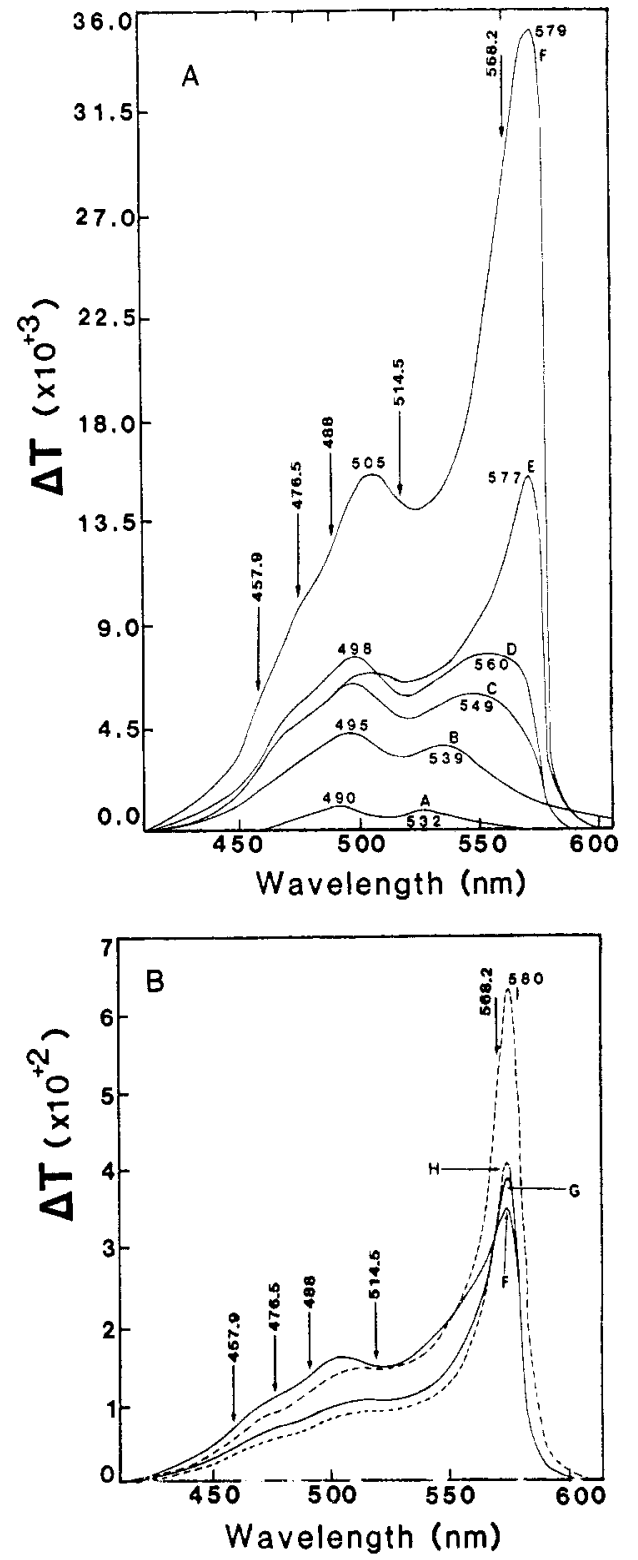

Figure 3. Electronic spectra of $S-120$ in lipid matrix (arachidic acid: methyl arachidate $9: 1$ ) at varying dye concentrations: A, 1\%; B, 4\%; C, $6.25 \%$; D, $7.7 \%$; E, 9.0\%; F, 11.8\%, G, $14.3 \%, \mathrm{H}, 16.7 \% ; \mathrm{I}, 18.2 \%$. In Figure $3 \mathrm{~A}$ the ordinate scale is factor of 10 lower than that of Figure $3 \mathrm{~B}$.

arated areas of $\mathrm{Ag}$ that are randomly distributed on the glass. Scanning electron micrographs of these preparations show that the films are composed of $\mathrm{Ag}$ particles ranging between 200 and $400 \AA .44,45$ The islands increase in size and eventually merge as the thickness of the film is increased. Contact between individual particles contributes to the tailing observed in the absorption spectrum. Figure 4 shows spectra of two different film preparations (dotted lines $B$ and D). Spectrum $B$ has an absorbance maximum near $490 \mathrm{~nm}$, whereas in $D$ the maximum is at ca. 540 $\mathrm{nm}$. The absorption band in D is much broader than in B and tails considerably to the red. Thus, it is apparent that the film in $\mathrm{D}$ contains larger particles, some of which are in contact. Spectra A and C (solid lines) were measured following transfer of a monolayer of S-120 (33.3 and $16.7 \mathrm{~mol} \%$ dye, respectively) onto the Ag-coated glass slides. In the case of spectrum A, the dye overlayer produces a splitting in the composite spectrum, with a minimum near $530 \mathrm{~nm}$ and a shoulder near $560 \mathrm{~nm}$. In the case of spectrum $C$, the composite spectrum is broad and shifted to the red of the $\mathrm{Ag}$ film. In both cases, spectra of the composite

(44) Schlegel, V.; Cotton, T. M., unpublished results.

(45) Uphaus, R. A.; Cotton, T. M.; Möbius, D. Thin Solid Films 1985 , 132,173 . 


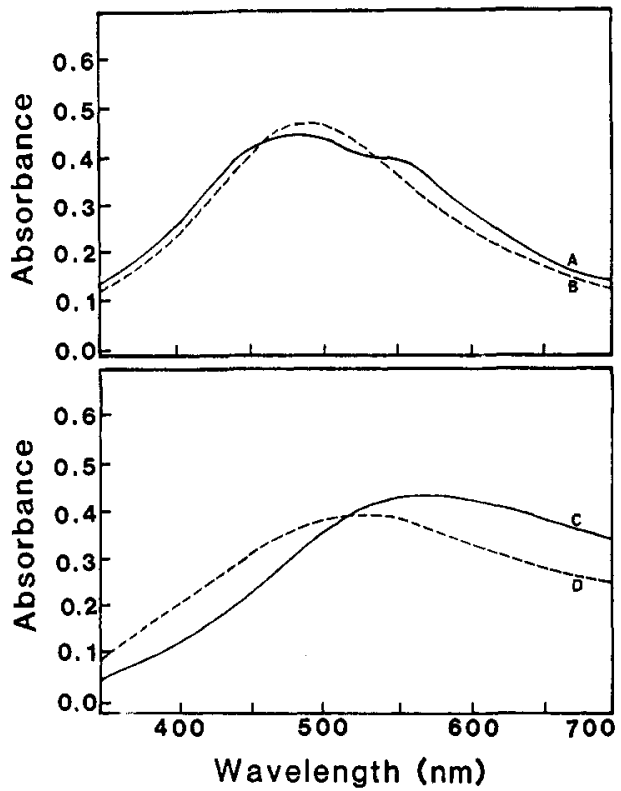

Figure 4. Electronc absorption spectra of the $\mathrm{Ag}$ films (dotted lines $\mathrm{B}$ and $\mathrm{D}$ ) and of the composite dye monolayer-Ag films (solid lines A (33.4 $\mathrm{mol} \%$ ) and $C(16.7 \mathrm{~mol} \%)$ ). In all spectra, the Ag film thickness was $50 \AA$. The spectral differences between the top frame and the bottom are due to different batches of $\mathrm{Ag}$ film.

films are not simply the sum of the film and dye overlayers.

These results are in accord with previous experimental results ${ }^{19-21}$ as well as theory. ${ }^{4,23,24}$ Glass et al. ${ }^{19}$ have shown that the absorption spectrum of rhodamine $\mathrm{B}$ and Nile blue on a $\mathrm{Ag}$ island film is not the sum of the dye and Ag spectra. Rather, the spectrum shows a splitting, as observed here in spectrum $A$. The spectral perturbation is greatest when the plasmon resonance of the $\mathrm{Ag}$ film is close to the absorption maximum of the adsorbate.

The optical response of the composite films is important with regard to the relative contributions of resonance and surface enhancement to the SERRS spectrum. The magnitude of the surface enhancement is determined from a comparison of the SERRS and RR excitation profiles, as discussed later. For the present purposes, it should be noted that the the laser excitation lines used in this study are at $457.9,476.5,488.0,514.5$, and 568.2 $\mathrm{nm}$. These are indicated in Figure 3.

SERRS Spectra of S-120 as a Function of Surface Coverage. The effect of dye concentration in the lipid matrix on the SERRS intensity was examined through the concentration range from 0.5 to $33.3 \mathrm{~mol} \%$. Figure 5 illustrates spectra obtained for a number of concentrations by using $457.9-\mathrm{nm}$ excitation. This wavelength is close to the high-energy shoulder on the major electronic transition at $522 \mathrm{~nm}$ in the monomeric dye spectrum. There are three important points to be noted from examination of Figure 5. First, under the experimental conditions employed, the detection limit for the dye is less than $0.5 \mathrm{~mol} \%$. This limit is even lower at wavelengths more closely in resonance to the absorption maximum of the dye. However, the dye is photosensitive and is not as stable when irradiated at longer wavelengths. The photostability and the effect of excitation wavelength on the spectrum will be discussed further below. Second, the band frequencies and relative intensities are independent of surface coverage. This indicates that there are no significant changes in the ground- or excited-state properties of the dye as a function of concentration. Such changes might be expected as effects of aggregation. The same concentration behavior was observed for excitation wavelengths close to the J-aggregate maximum $(568.2 \mathrm{~nm})$. Third, the band frequencies and intensities of the dye on the surface are identical with its solution counterparts. Thus, there is no strong interaction between the dye and Ag; no significant contribution from a chemical enhancement mechanism is present in these systems. This is in contrast to previous studies of $S-120$ on silica, in which spectral changes were indicative of dye interactions with the surface. ${ }^{46}$

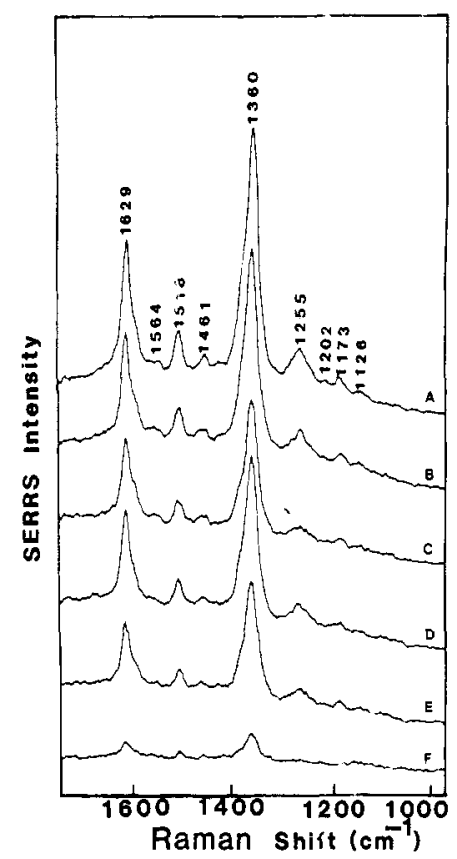

Figure 5. SERRS spectra of S-120 dye monolayers on Ag island films $(50 \AA)$. Monolayer composition (mole percent in lipid matrix) is as follows: A, 20; B, 12.5; C, 9.1; D, 6.25; E, 3.2; F, 0.5. Experimental conditions: laser excitation wavelength, $457.9 \mathrm{~nm}$; power $6 \mathrm{~mW}$; integration time, $1 \mathrm{~s} ; 30$ scans.
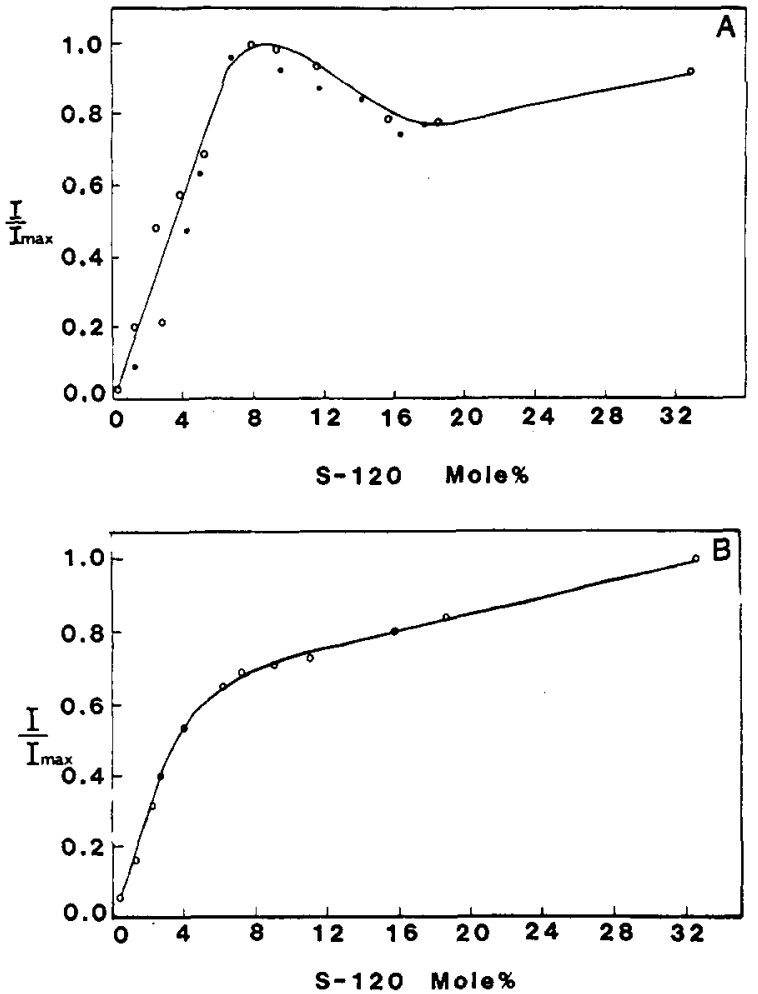

Figure 6. Intensity of the SERRS band at $1360 \mathrm{~cm}^{-1}$ versus mole percent of dye in lipid matrix. Intensities have been normalized to the maximum value. Part $\mathrm{A}$ is for data acquired with one scan. The solid and dotted lines correspond to two different batches of $\mathrm{Ag}$ island films. The solid dots correlate with electronic absorption spectra shown in Figure 4, curve A. The open dots correlate with electronic absorption spectra shown in Figure 4, curve C. Part B is for data acquired by adding 30 scans.

The relationship between dye concentration and SERRS intensity for spectra recorded during $1 \mathrm{~s}$ is shown in Figure 6A. The ordinate is normalized with respect to the maximum intensity.

(46) Rabolt, J. F.; Schlotter, N. E.; Swalen, J. D.; Santo, R. J. Polym. Sci. $1983,2 I, 1$. 
As may be seen, the signal intensity rises rapidly between 0.5 and $9 \mathrm{~mol} \%$, following which it slowly decreases as the coverage is increased to $16 \mathrm{~mol} \%$. A slight increase in intensity occurs between 16 and $33.3 \mathrm{~mol} \%$. At $33.3 \mathrm{~mol} \%$, the Ag film surface is essentially completely covered with dye. The lipid matrix is adsorbed on top of the dye rings as determined from the surface isotherm measurements, i.e., the apparent area per molecule is equal to the area of the dye molecule. This configuration (two molecules of lipid per one molecule of dye) confers the maximum interaction between hydrocarbon tails. At this coverage, the signal intensity stabilizes at about $70-80 \%$ of the maximum value.

The general shape of the concentration vs intensity curve was highly reproducible and was observed for several series of slides prepared at different times. However, the absolute intensities did vary somewhat with film preparation. Thus, the open circles correspond to data obtained with the film exhibiting the absorption spectrum shown in Figure 4A, whereas the solid circles correspond to that in Figure $4 C$. The magnitude of the enhancement was 2 -fold greater for film A. Therefore, the intensities in film B were multiplied by 2 to display the concentration dependence in a single figure.

Photostability of S-120 Monolayers on Ag Island Films. The time dependence of the scattering intensity for each of the different films was monitored between 1 s (i.e., spectrum recorded immediately) and $8 \mathrm{~min}$. An advantage to the use of the diode array is that the entire spectrum may be obtained in $1 \mathrm{~s}$. The signal strength was observed to decrease exponentially with time and reach a steady state value after $8 \mathrm{~min}$. The concentration dependence for samples irradiated for $30 \mathrm{~s}$ is shown in Figure 6B. The signal intensity does not maximize at $9 \mathrm{~mol} \%$ under these conditions. Rather, it increases rapidly with concentrations between 0 and $4 \mathrm{~mol} \%$ and then increases more slowly with higher dye concentrations.

$R R$ and SERRS Excitation Profiles. The RR excitation profile for $S .120$ in methanol at several different concentrations (not shown) was found to follow the electronic absorption spectrum, as expected. Figure 7A shows the excitation profile for the monolayer containing $33.3 \mathrm{~mol} \mathrm{\%}$ of the dye on the $\mathrm{Ag}$ film. The upper curve is for the $1360-\mathrm{cm}^{-1}$ band intensity, and the lower one is for the $1629-\mathrm{cm}^{-1}$ band. The intensities of the peaks are normalized for the same laser power $(6 \mathrm{~mW})$ and slit settings at the different excitation wavelengths. A correction for the in strument throughput and detector response was made by referencing the spectra to methanol at the same excitation wavelengths. Although these are not high-resolution profiles, it is obvious that the SERRS intensity for both bands is maximal at approximately $514.5 \mathrm{~nm}$, or close to the shoulder on the J-aggregate band. This is also close to the plasmon resonance of the Ag film. It is surprising that the enhancement is not maximal at $568.2 \mathrm{~nm}$ or closer to the absorbance maximum of the J-aggregate. This indicates that the spectral intensity is controlled by the optical properties of the film and not the molecular resonance.

To obtain an estimate of the EM enhancement factor, it is necessary to determine the RR intensity as a function of the number of scatterers in the laser beam. Therefore, the RR intensity of two bands were measured for S-120 at five different concentrations in methanol $\left(2.5 \times 10^{-4}, 1.22 \times 10^{-5}, 2.48 \times 10^{-6}\right.$, $8.25 \times 10^{-7}$, and $\left.4.06 \times 10^{-8} \mathrm{M}\right)$. The effective path length or laser penetration depth at different excitation wavelengths was determined by the procedure of Shriver and Dunn. ${ }^{47}$ From the concentration isotherm, the mean concentration $(\bar{c})$ at $I / I_{\max }=$ 0.5 was calculated. This value is then used to determine $b$, the penetration depth:

$$
b=\frac{0.301}{\bar{c}\left(E_{\mathrm{ex}}+E_{\mathrm{RS}}\right)}
$$

where $E_{\mathrm{ex}}$ and $E_{\mathrm{RS}}$ are the extinction coefficients at the laser excitation and Raman-shifted wavelengths, respectively. With the value obtained for $b$, it is then possible to determine the number

(47) Shriver, D. F.; Dunn, J. B. R. Appl. Spectrosc. 1974, 28, 319.
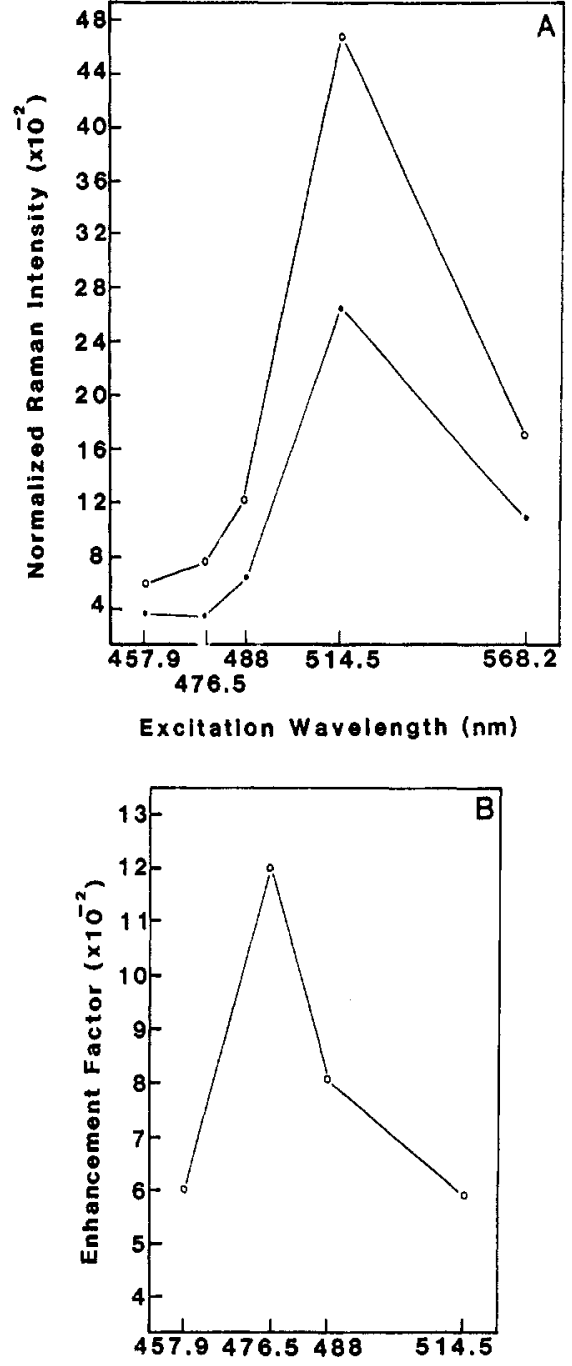

Excitatlon Wavelength $(\mathrm{nm})$

Figure 7. SERRS excitation profiles (A) and enhancement factor as a function of excitation wavelength (B) of S-120 monolayer (33.3\% in lipid matrix). In part $A$, the open circles are data points for the $1360-\mathrm{cm}^{-1}$ band, and the solid circles are data points for the $1629-\mathrm{cm}^{-1}$ band. Experimental conditions were as follows: laser excitation wavelengths, $457.9,476.5,488,514.5$, and $568.2 \mathrm{~nm}$; laser power, $6 \mathrm{~mW}$; integration time, $1 \mathrm{~s} ; 30$ scans.

of molecules that contribute to RR scattering at each wavelength.

Finally, the surface enhancement factor (EF) is calculated from the ratio of the Raman scattering intensity for an equal number of molecules on the surface $\left(N_{\mathrm{Ag}}\right)$ and in solution $\left(N_{\mathrm{soln}}\right)$ :

$$
\mathrm{EF}=\frac{I_{\text {SERRS }} / N_{\mathrm{Ag}}}{I_{\mathrm{RR}} / N_{\text {soln }}}
$$

The EF does not include the resonance contribution since it is present in both the numerator and denominator terms.

A second method was also used to calculate EFs. The RR spectrum of 30 monolayers containing $16.67 \mathrm{~mol} \%$ dye was measured. The SERRS intensity at a given wavelength was divided by the RR intensity for an equal number of molecules. This method gave a slightly lower value for EF ( 430 at $457.9-\mathrm{nm}$ excitation, as compared to 608 by using the above procedure). A plot of the calculated EF as a function of excitation wavelength is shown in Figure 7B. The maximum is near $476.5 \mathrm{~nm}$, with some indication of a shoulder near $490 \mathrm{~nm}$. It is notable that the EF is greatest near the maximum in the Ag island film (Figure 4A) and near the minimum in the excitation profile for the RR spectrum of the dye. The same EF vs excitation wavelength plot was also observed for the slides that exhibited the absorption spectrum shown in Figure 4C. 
The maximum enhancement factor $\left(1.2 \times 10^{3}\right)$ shown in this plot is similar to the value observed for other resonantly enhanced molecules. ${ }^{16,44}$ Siiman et al. ${ }^{48}$ showed that SERRS scattering from DABS-ASP on silver hydrosols was enhanced by only 3 orders of magnitude over the RR intensity. Weitz et al. ${ }^{17}$ showed that the lower enhancement for RR scattering is consistent with a model that considers the relative rates of excitation and deactivation of the molecular resonance at the metal particle surface.

\section{Discussion}

The relationship between the mole percent of S-120 dye present in the lipid monolayer and the SERRS intensity of the dye is complex, as shown in Figure 6. SERRS intensities obtained with 1.0-s integration time (Figure 6A) rise approximately monotonically for dye concentrations between 0.5 and $9 \mathrm{~mol} \%$. Above $9 \mathrm{~mol} \%$ a gradual decrease in signal intensity is observed with increasing concentration to ca. $16 \mathrm{~mol} \%$. The overall decrease in signal is about $20 \%$ of the maximum value. At higher surface coverages, the SERRS intensity appears to increase slightly. Identical intensity vs coverage behavior was observed for many different batches of $\mathrm{Ag}$ films, including films that exhibited weaker overall enhancement because of differences in the surface morphology.

There are at least two possible explanations for the coverage dependence observed here. First, the maximum in the SERRS intensity may reflect the onset of J-aggregate formation. Second, the data may support theoretical models ${ }^{3-5,16}$ that predict a maximum in the signal intensity at submonolayer coverages as a result of changes in the dielectric properties of the overlayer and/or dipole-dipole coupling. Both of these possibilities are addressed below.

As can be seen from the spectra shown in Figure 3, the formation of a significant concentration of $J$-aggregate occurs at dye coverages near $9 \mathrm{~mol} \%$, or precisely where the SERRS intensity is maximal. Consequently, the decrease in monomer absorbance near $522 \mathrm{~nm}$ may account for the decrease in SERRS intensities at higher dye coverages. However, on comparing spectra $\mathrm{A}-\mathrm{I}$ in Figure 3, it can be seen that the J-aggregate has a shoulder at $510 \mathrm{~nm}$ which increases with increasing $\mathrm{J}$-aggregate concentration in the film. Hence, there is no net decrease in absorbance as the dye coverage increases. Also, plots of signal intensity vs dye coverage at various excitation wavelengths below $514.5 \mathrm{~nm}$ show the same general shape. That is, the signal increases with surface coverage and then decreases rapidly at a certain coverage (18.2\%). At $514.5 \mathrm{~nm}$, the signal was maximal at a much lower coverage $(9 \mathrm{~mol} \%)$. If the SERRS intensity is dependent solely upon absorbance, as is the case for RR enhancement, it should increase with optical density at all excitation wavelengths. Obviously, the relationship is more complex. Coupling between the dye absorption and the $\mathrm{Ag}$ plasmon resonance strongly influences the wavelength dependence of the SERRS. Near $514.5 \mathrm{~nm}$, the composite dye-Ag film spectrum (Figure 4A) has a weak minimum, indicating that the damping of the plasmon resonance is maximal at this wavelength. Hence, the SERRS intensity should be most sensitive to the dielectric properties (or the surface coverage) of the dye layer at this wavelength. In summary, it appears that the RR contribution alone does not dominate the response in this region of the spectrum. Both RR and surface enhancement contribute to the strong SERRS intensities.

Further support for the dielectric effect on the SERRS intensity may be found in the relationship between the SERRS intensity vs absorbance at $568.2 \mathrm{~nm}$ (or closer in resonance to the J-aggregate). A gradual increase in intensity occurs with absorbance up to $18 \mathrm{~mol} \%$, at which point a sharp increase occurs. The RR contribution is more significant at this excitation wavelength at low dye coverages. At higher coverages (above $18 \mathrm{~mol} \%$ ), a red shift in the composite J-aggregate-silver film spectrum occurs. The rapid increase in SERRS intensity at this dye concentration suggests that some EM enhancement is present.

(48) Siiman, O.; Lepp, A.; Kerker, M. J. Phys. Chem. 1983, 87, 5319.
The above results support the proposed dipole-dipole or effective medium models ${ }^{3-5.16}$ that have been proposed to account for the coverage dependence. It is not possible to distinguish between the models on the basis of data presented here. The formation of $\mathrm{J}$-aggregate is expected to both increase the dipole-dipole coupling and strongly perturb the dielectric properties of the overlayer. The discontinuity near $9 \%$ suggests that the dipoledipole coupling, which is dependent upon $d^{-3}$ (where $d$ is the distance between dye molecules) becomes significant. As $d$ decreases to values less than $4 a$ (where $a$ is the molecular radius) dipole-dipole coupling leads to a decrease in the scattering intensity. Indeed, direct contact between dye molecules occurs at higher concentration where dimer and aggregate formation is significant. Theory predicts that the Raman intensity should decrease by ca. $15 \%$ as the intermolecular distance decreases to zero. This value is very close to that observed.

Finally, the photostability of the dye monolayer is related to the surface coverage. Figure $6 \mathrm{~A}$ illustrates the effect of surface coverage on SERRS intensity for 1-s integration periods. Figure $6 \mathrm{~B}$ represents the results obtained for the sum of 30 spectra, each of which represents a 1-s integration period. The coverage dependence is obviously much different for the two data sets. The SERRS intensity for a single scan maximizes at ca. $9 \mathrm{~mol} \%$, whereas for 30 scans the maximum is near $20 \mathrm{~mol} \%$. No peak is observed in the latter curve; the signal increases in an approximately linear fashion to about $4 \mathrm{~mol} \%$. The differences between results obtained with 1 scan and 30 scans can be explained in terms of the greater photobleaching of the dye molecules between the Ag islands during prolonged irradiation, as noted by Garoff et al. ${ }^{31,32}$ Also, a preferential bleaching of the monomer is expected because of the relatively long lifetime of the monomer as compared to the $\mathrm{J}$-aggregate. The signal intensity decreases exponentially with exposure time and approaches a steady-state value after ca. $8 \mathrm{~min}$. Multiple scan data can be considered equivalent to a hole-burning experiment. Dye molecules between the islands (as well as monomer species) are preferentially bleached, and dipole-dipole coupling is reduced. Due to the lower effective dye concentration, the peak intensity is less than for shorter irradiation periods and increases more slowly with increasing concentration. At sufficiently high concentration, the multiple-scan curve approaches the single-scan curve, as expected.

The relationship between SERRS intensity and coverage dependence reported here is quite different from that of Zeman et al. ${ }^{5}$ and Hildebrandt and Stockburger. ${ }^{18}$ To establish possible reasons for the differences, it is necessary to consider the experimental factors that contribute to the coverage dependence. These include the following: (1) Ag particle size and shape; (2) dielectric medium; (3) proximity of the Ag plasmon frequency to strong absorption bands in the adsorbate; (4) SERRS enhancement mechanism(s). In the following discussion, each of these factors is considered for the three systems.

There are undoubtedly considerable differences in the size and shape of $\mathrm{Ag}$ particles that are vacuum-deposited on glass as compared to sols. The latter are spherical, and several theories $5,15,24$ predict that the relationship between surface coverage and SERRS intensity is quite sensitive to the aspect ratio of Ag spheroids. Particles with smaller aspect ratio, or more spherical in shape, are predicted to exhibit maximum SERRS intensities at higher coverages in the treatment of Zeman et al. ${ }^{5}$ (e.g., the $1544-\mathrm{cm}^{-1}$ band of $\mathrm{COPc}$ showed a continuous increase in SERRS intensity up to 0.5 monolayer for a $3: 1$ ratio of major/minor axis, whereas the intensity maximized at $0.06 \%$ for an aspect ratio $5: 1$ ). Thus, Ag sols may be less sensitive to surface coverage effects. This certainly appears to be the case on the basis of the published data. There may also be significant differences in the Ag island film structures prepared in different laboratories because of variations in the deposition procedures.

The differences between the dielectric medium of the $\mathrm{Ag}$ particles is quite significant (air in the experiments of Zeman et al., ${ }^{5}$ water in the Ag sols, ${ }^{18}$ and hydrocarbons in our monolayer experiments). However, we have not observed a large effect on the enhancement factor when the spectra of the dye-coated Ag 
films were collected in the presence of water. One important difference is that the island films contain dye molecules between the islands at approximately the same concentration as on the surface. This could affect the coupling between metal particles. In the case of sols, the dye solution is very dilute, although aggregation of the $\mathrm{Ag}$ particles does occur to some extent. In fact, aggregation is thought to be essential for observation of SERRS in colloidal Ag systems. Nevertheless, coupling between particles might be significantly lower in the case of sols.

A comparison of the three experimental systems shows there are differences in the proximity of the $\mathrm{Ag}$ plasmon resonance to the absorption band of the adsorbate (factor 3 ). CoPc was excited very close to resonance $(647 \mathrm{~nm})$ with the electronic transition $(650 \mathrm{~nm})$, but no spectrum was given for the Ag film. ${ }^{5}$ Thus, it is not possible to determine the overlap between the molecular and plasmon resonance. In the rhodamine $6 \mathrm{G}$ case, the absorption maximum was at $530 \mathrm{~nm}$ and the sol absorption was at $415 \mathrm{~nm}$, with tailing to the red. ${ }^{18}$ SERRS spectra were excited at 514.5 $\mathrm{nm}$, which is not strongly in resonance with either the sol or the dye. Loss of EM enhancement because of the dye absorbance might be expected to be minimal under these conditions. In contrast, the overlap between S-120 absorption and the plasmon resonance is considerable in our experiments.

The fourth factor concerns the origin of the enhancement. There are three possible contributions: EM, resonance Raman, and chemical enhancement. Both EM and RR enhancement are present in the CoPc system. It is not possible to tell whether there is a chemical component, however, since a complete description of band frequencies was not given ${ }^{5}$ for the SERRS or RR spectrum. There is evidence for band intensity differences with surface coverage (submonolayer to multilayer), which suggests that there is a strong interaction between the first layer and the $\mathrm{Ag}$. In the case of the rhodamine $6 \mathrm{G}$ study, all three components appear to be present, at least when certain anions are added to the system. Special sites are involved in the additional SERRS enhancement observed in the presence of $\mathrm{Cl}^{-}$. Although the concentration of these sites is very low (only 3.3 sites per Ag particle), the SERRS enhancement increased by an additional factor of 70 over classical EM enhancement when $\mathrm{Cl}^{-}$was added to the sol. The experimental data (vibrational spectrum and absorption energy) support chemisorption of rhodamine $6 \mathrm{G}$ to the $\mathrm{Ag}$ particle.

Another possible explanation for differences between the three systems involves the methodology used to vary the surface coverage. In our experiments, LB monolayers allow precise control over the distribution of the dye molecules on the Ag film. Furthermore, the lipid matrix not only maintains a more uniform intermolecular dye distance but also minimizes site-specific adsorption of the dye on $\mathrm{Ag}$. These two factors are not as easily controlled in either the vacuum deposition ${ }^{5}$ or $\mathrm{Ag} \mathrm{sol}^{18}$ experiments. In the experiments of Zeman et al. vacuum deposition was used to vary the concentration of $\mathrm{CoPc}$ on $\mathrm{Ag} .^{5}$ Although the surface coverage was determined following the SERRS experiments, the distribution of the dye molecules is not known (e.g., clumps or aggregates of the $\mathrm{CoPc}$ molecules may well be present even at low coverage). In the monolayer systems the dye molecules are randomly distributed on the surface; there is no opportunity for the initial saturation of specific sites at low dye coverage. Therefore, the measured SERRS intensity represents the averge of that resulting from all of the various types of surface sites. However, our experiments are complicated by the tendency of S-120 to form J-aggregates. In subsequent studies, a nonaggregating, amphiphilic porphyrin will used to determine whether the same type of concentration dependence is seen for all resonantly enhanced molecules having absorption bands close to the plasmon resonance of the $\mathrm{Ag}$ island films.

\section{Conclusions}

The LB monolayer studies discussed above have established the following points:

1. The absorption or transmission spectrum of the composite S- 120 monolayer/Ag films is indicative of coupling between the dye absorption and the $\mathrm{Ag}$ plasmon resonance.

2. The transmittance spectra of the dye-containing monolayers on glass slides show a sharp increase in J-aggregate structure at concentrations near 9 mol \%.

3. The SERRS intensity maximizes near $9 \mathrm{~mol} \%$, or close to the surface coverage at which J-aggregate concentration becomes significant.

4. The photochemical response of the dye monolayers on $\mathrm{Ag}$ indicates that dye molecules adsorbed on glass are bleached, whereas those on the $\mathrm{Ag}$ are not.

5. SERRS spectra obtained for the entire range of dye coverages show no evidence (band shifts or intensity differences) for strong chemical interactions between the dye and the Ag.

6. The SERRS excitation spectrum does not follow the absorption spectrum of S-120 nor does it change substantially with dye coverage, indicating a strong contribution from EM enhancement.

7. The wavelength dependence of the EF follows the Ag plasmon spectrum. The surface enhancement contributes $\mathrm{ca} .10^{3}$ to the signal intensity at excitation wavelengths near $500 \mathrm{~nm}$.

The data support a significant electromagnetic enhancement contribution to the overall SERRS intensities. Experimental observations of the effect of dye coverage on SERRS intensity agree with theoretical predictions based upon changes in the effective dielectric medium or increased dipole-dipole coupling between dye molecules with increasing dye coverage. Additional experimental data is needed to distinguish between the latter two effects.

Acknowledgment. The financial support of the U.S. Department of Energy, Chemical Sciences Division (DE-FG0284ER 13261 ), is gratefully acknowledged. We thank W. Zeiss and G. Debuch for technical assistance in monolayer preparation.

Registry No. S-120, 67878-03-9; Ag, 7440-22-4; arachidic acid, 506-30-9; methyl arachidate, 1120-28-1. 